\title{
WISE morphological study of Wolf-Rayet nebulae
}

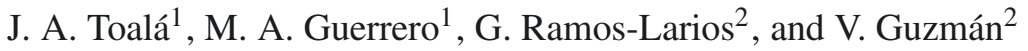 \\ ${ }^{1}$ Instituto de Astrofísica de Andalucía, IAA-CSIC, Glorieta de la Astronomía s/n, 18008 Granada, Spain \\ e-mail: toala@iaa.es \\ 2 Instituto de Astronomía y Meteorología, Av. Vallarta No. 2602, Col. Arcos Vallarta, CP 44130, 2602 Guadalajara, Jalisco, Mexico
}

Received 21 January 2015 / Accepted 23 March 2015

ABSTRACT

\begin{abstract}
We present a morphological study of nebulae around Wolf-Rayet (WR) stars using archival narrow-band optical and Wide-field Infrared Survey Explorer (WISE) infrared images. The comparison among WISE images in different bands and optical images proves to be a very efficient procedure to identify the nebular emission from WR nebulae, and to disentangle it from that of the ISM material along the line of sight. In particular, WR nebulae are clearly detected in the WISE W4 band at $22 \mu \mathrm{m}$. Analysis of available mid-IR Spitzer spectra shows that the emission in this band is dominated by thermal emission from dust spatially coincident with the thin nebular shell or most likely with the leading edge of the nebula. The WR nebulae in our sample present different morphologies that we classified into well defined WR bubbles (bubble $\mathcal{B}$-type nebulae), clumpy and/or disrupted shells (clumpy/disrupted $C$-type nebulae), and material mixed with the diffuse medium (mixed $\mathcal{M}$-type nebulae). The variety of morphologies presented by WR nebulae shows a loose correlation with the central star spectral type, implying that the nebular and stellar evolutions are not simple and may proceed according to different sequences and time-lapses. We report the discovery of an obscured shell around WR 35 only detected in the infrared.
\end{abstract}

Key words. stars: massive - stars: Wolf-Rayet - stars: winds, outflows - ISM: bubbles

\section{Introduction}

Massive stars $\left(M_{\mathrm{i}} \gtrsim 30 M_{\odot}\right)$ end up their lives as WolfRayet(WR) stars. Before entering the WR phase, these stars evolve through the red or yellow supergiant (RSG or YSG) or luminous blue variable (LBV) phases, depending on the initial stellar mass, and eject their envelopes via copious slow winds expanding at $10-100 \mathrm{~km} \mathrm{~s}^{-1}$. When a fast stellar wind (1000-2000 $\mathrm{km} \mathrm{s}^{-1}$ ) develops during the WR phase, it sweeps up the previously ejected slow RSG or LBV wind material to form a WR nebula. The swept up circumstellar material is photoionized by the central star and has temperatures of $\sim 10^{4} \mathrm{~K}$.

These WR nebulae present different morphological features such as bubbles, blowouts, clumps, filaments, and diffuse emission. These nebulae are observable at all frequencies of the electromagnetic spectrum: radio (e.g. Arnal \& Cappa 1996; Arnal et al. 1999; Cappa et al. 2002, 2008, 2009), infrared (IR; e.g. van Buren \& McCray 1988; Gvaramadze et al. 2010a; Mauerhan et al. 2010; Wachter et al. 2010, 2011; Flagey et al. 2011; Stringfellow et al. 2012), optical (e.g. Chu 1982; Treffers \& Chu 1982a; Chu et al. 1983; Gruendl et al. 2000; Stock \& Barlow 2010; Fernández-Martín et al. 2012), and X-rays (e.g. Bochkarev 1988; Wrigge et al. 1994; Wrigge 1999; Chu et al. 2003; Wrigge \& Wendker 2002; Wrigge et al. 2005; Zhekov \& Park 2011; Toalá et al. 2012, 2015).

Chu (1981) started a series of works proposing a coherent morphological classification of nebulae associated with WR stars as $R$ - radiatively excited H II regions, $E$ - stellar ejecta, and $W$ - wind-blown bubbles (see Chu 2003, for an updated review of the morphology of WR nebulae). The $R$-type

\footnotetext{
$\star$ Appendix $\mathrm{A}$ is available in electronic form at http://www. aanda.org
}

nebulae present optical emission lines with widths comparable to those in ordinary $\mathrm{H}$ II regions, and are further divided into amorphous $\mathrm{H}$ II regions $\left(R_{\mathrm{a}}\right)$ and shell-structured $\mathrm{H}$ II regions $\left(R_{\mathrm{S}}\right)$. It has been proposed that the $E$-type nebulae, characterized by their highly clumpy appearance and irregular velocity field, form through sudden episodes of mass ejection. Finally, the $W$-type nebulae present a thin sheet of gas and filaments curving around a WR star that is close to the geometric center of the nebula or offset toward the brightest rim. $W$-type nebulae are predicted by numerical simulations of the circumstellar medium (CSM) around WR stars (e.g. García-Segura et al. 1996a,b; Freyer et al. 2003, 2006; Toalá \& Arthur 2011). Using this classification scheme, Stock \& Barlow (2010) extended and revised the morphologies of a sample of southern WR nebulae.

Chu (1981) found a correlation, later confirmed by Chu et al. (1983), between nebular morphology and spectral type of the central WR star: WC stars are mostly associated with $R_{\mathrm{S}}$ nebulae, whereas the central stars of $W$ nebulae are mostly early WN stars and those of $E$ nebulae are exclusively of spectral type WN8. This correlation was interpreted in terms of nebular evolution, so that $W$ nebulae, at an early nebular phase, will precede $R_{\mathrm{S}}$ nebulae (Chu et al. 1983).

The classification scheme described above is very demanding observationally. It requires both direct line imaging and spectroscopic campaigns to investigate the morphology, kinematics, and abundances of the ionized material in order to disentangle the emission of the WR nebulae from that of the interstellar medium (ISM). In recent years, mid-IR observations have proved very useful for studying WR nebulae or for discovering new nebulae around evolved stars (e.g. Wachter et al. 2010). In particular, Spitzer observations in the $24 \mu \mathrm{m}$ band have been found to be sensitive to the WR nebular emission, whereas the 
Table 1. Stellar and nebular parameters of the WR sample.

\begin{tabular}{|c|c|c|c|c|c|c|c|c|c|c|}
\hline WR & Nebula $^{a}$ & RA & Dec & $\begin{array}{c}\text { Galactic Coord. } \\
\qquad \begin{array}{c}l, b \\
\left({ }^{\circ}\right) \\
\end{array}\end{array}$ & $\begin{array}{c}d \\
(\mathrm{kpc}) \\
\end{array}$ & $\begin{array}{c}z \\
(\mathrm{pc}) \\
\end{array}$ & $\begin{array}{l}\text { Spectral } \\
\text { Type }\end{array}$ & $\begin{array}{c}v_{\infty} \\
\left(\mathrm{km} \mathrm{s}^{-1}\right) \\
\end{array}$ & $\begin{array}{c}\text { Optical } \\
\text { Observation }^{b}\end{array}$ & $\begin{array}{c}\text { Nebular } \\
\text { Type }\end{array}$ \\
\hline 6 & S 308 & 065413.05 & -235542.1 & $234.76-10.08$ & 1.50 & -262 & WN4-s & 1700 & CTIO (H $\alpha,[\mathrm{O}$ III] $])$ & $\mathcal{B}$ \\
\hline 7 & NGC 2359 & 071829.13 & -131301.5 & $227.75-0.13$ & 3.67 & -8 & WN4-s & 1600 & SuperCOSMOS/DSS & $\mathcal{B}$ \\
\hline 8 & & 074458.22 & -315429.6 & $247.07-3.79$ & 3.47 & -229 & WN7/WCE+? & 1590 & SuperCOSMOS & C \\
\hline 16 & Anon & 095452.91 & -574338.3 & $281.08-2.55$ & 2.37 & -105 & WN8h & 650 & SuperCOSMOS & $\mathcal{B}$ \\
\hline 18 & NGC 3199 & 101702.28 & -575446.9 & $283.57-0.97$ & 2.20 & -37 & WN4-s & 1800 & DSS & $C$ \\
\hline 22 & Anon & 104117.52 & -594036.9 & $287.17-0.85$ & 3.24 & -48 & $\mathrm{WN} 7 \mathrm{~h}+\mathrm{O} 9 \mathrm{III}-\mathrm{V}$ & 1785 & DSS & $\mathcal{M}$ \\
\hline 23 & Anon & 104138.33 & -584618.8 & $286.78-0.03$ & 3.24 & -2 & wC6 & 2340 & DSS & $\mathcal{B}$ \\
\hline 30 & Anon & 105106.01 & -621701.8 & $284.44-2.61$ & 5.83 & -263 & WC6+O6-8 & 2100 & SuperCOSMOS & $\mathcal{M}$ \\
\hline $31 \mathrm{a}$ & & 105359.66 & -602644.3 & $288.94-0.81$ & 8.0 & -113 & WN11h & 365 & SuperCOSMOS & $\mathcal{B}$ \\
\hline 35 & Anon & 110022.10 & -611351.0 & $289.97-1.19$ & 17.87 & -371 & WN6h-w & 1100 & SuperCOSMOS & $C$ \\
\hline $35 \mathrm{~b}$ & Anon & 110002.30 & -601401.0 & $289.63-0.24$ & 2.19 & -9 & WN4 & & SuperCOSMOS & $\mathcal{M}$ \\
\hline 38 & Anon & 110546.52 & -611349.1 & $290.57-0.92$ & 5.83 & -94 & WC4 & 3200 & SuperCOSMOS & $\mathcal{M}$ \\
\hline 40 & RCW 58 & 110617.21 & -653035.2 & $292.31-4.83$ & 2.26 & -190 & WN8h & 650 & $\mathrm{CS}(\mathrm{H} \alpha,[\mathrm{O} \mathrm{III}])$ & C \\
\hline 52 & Anon & 131828.00 & -580813.6 & $306.50+4.54$ & 1.51 & 120 & WC4 & 3225 & SuperCOSMOS & $\mathcal{M}$ \\
\hline 54 & Anon & 133243.79 & -650127.9 & $307.27-2.50$ & 7.53 & -321 & WN5-w & 1500 & SuperCOSMOS & $\mathcal{M}$ \\
\hline 55 & RCW 78 & 133330.13 & -621901.2 & $307.80+0.16$ & 6.03 & 17 & WN7 (WNE-w) & 1200 & SuperCOSMOS & C \\
\hline 68 & G320.5-1.4 & 151821.0 & -593810.0 & $320.54-1.88$ & 3.27 & -107 & WC7 & 2100 & SuperCOSMOS & $\mathcal{M}$ \\
\hline 75 & RCW 104 & 162426.23 & -513206.1 & $332.84-1.48$ & 2.18 & -56 & WN6-s & 2300 & $\mathrm{CS}(\mathrm{H} \alpha,[\mathrm{O} \mathrm{III}])$ & $\mathcal{B}$ \\
\hline 85 & RCW 118 & 171427.13 & -394547.0 & $347.43-0.61$ & 4.66 & -50 & WN6h-w (WNL) & 1400 & SuperCOSMOS & $C$ \\
\hline 86 & RCW 130 & 171823.06 & -342430.6 & $352.25+1.85$ & 2.86 & 92 & WC7(+B0III-I) & 1855 & DSS & $\mathcal{M}$ \\
\hline 94 & Anon & 173307.14 & -333823.7 & $354.60-0.25$ & 3.12 & -14 & WN5-w & 1300 & SuperCOSMOS & $\mathcal{M}$ \\
\hline 95 & & 173619.76 & -332610.9 & $355.13-0.70$ & 2.09 & -26 & WC9d & 1900 & SuperCOSMOS & $C$ \\
\hline 101 & Anon & 174509.10 & -315016.0 & $357.47-1.43$ & 3.18 & -79 & WC8 & & SuperCOSMOS & $C$ \\
\hline 102 & $\mathrm{G} 2.4+1.4$ & 174547.00 & -261029.0 & $2.38+1.41$ & 5.56 & 137 & WO2 & 5000 & SuperCOSMOS & $\mathcal{B}$ \\
\hline 113 & RCW 167 & 181907.36 & -113759.2 & $18.91+1.75$ & 1.79 & 55 & WC8d+O8-9IV & 1700 & DSS & $\mathcal{M}$ \\
\hline 116 & Anon & 182704.28 & -122252.3 & $19.16-0.32$ & 2.48 & -14 & WN8h & 800 & SuperCOSMOS & $C(\mathcal{M} ?)$ \\
\hline 124 & M1-67 & 191130.88 & +165138.2 & $50.20+3.31$ & 3.36 & 194 & WN8h & 710 & DSS & C \\
\hline 128 & Anon & 194832.20 & +181203.7 & $55.62-3.79$ & 9.37 & -619 & WN4(h)-w & 2050 & $\operatorname{MLO}(\mathrm{H} \alpha,[\mathrm{O} \mathrm{III}])$ & $\mathcal{B}$ \\
\hline 131 & & 200019.12 & +331551.1 & $69.90+1.71$ & 11.78 & 352 & WN7h & 1400 & DSS & $C$ \\
\hline 134 & Anon & 201014.20 & +361035.1 & $73.45+1.55$ & 1.74 & 47 & WN6-s & 1700 & $\operatorname{MLO}(\mathrm{H} \alpha,[\mathrm{O} \mathrm{III}])$ & $C$ \\
\hline 136 & NGC 6888 & 201206.55 & +382117.8 & $75.48+2.43$ & 1.26 & 53 & WN6(h)-s & 1600 & DSS (Red, Blue) & $\mathcal{B}$ \\
\hline
\end{tabular}

Notes. All parameters have been adopted from van der Hucht (2001), but the spectral classification and terminal velocities have been revised according to Hamann et al. (2006) and Sander et al. (2012). ${ }^{(a)}$ The name Anon is given to those nebulae that have not been catalogued as independent objects nebula, as those that are a small portion of a larger nebular region (see Chu 1981; Chu et al. 1983). ${ }^{(b)}$ CTIO: Cerro Tololo Inter-American Observatory, SuperCOSMOS: Super COSMOS Sky Survey, DSS: STScI Digitized Sky Survey, CS: Curtis-Schmidt, and MLO: Mount Laguna Observatory.

near-IR $K$ and mid-IR $8 \mu \mathrm{m}$ bands mainly trace the emission of material along the line of sight (e.g. Wachter et al. 2010; Stringfellow et al. 2012). Mid-IR observations of WR nebulae are thus a promising tool with which to study these objects.

Here we present Wide-field Infrared Survey Explorer (WISE; Wright et al. 2010) images of a sample of 31 WR stars and compare them with narrowband optical images (Sect. 2). This observational approach provides a straightforward method to for identifying nebulae around WR stars, clearly separating its emission from that of the ISM. The nature of the emission of WR nebulae in different WISE bands is investigated using mid-IR Spitzer spectra (Sect. 3). Their morphologies have been broadly grouped into three morphological types that can be interpreted in the framework of the evolution of the previously ejected dense wind through different evolutive paths (Sect. 4).

\section{Observations}

We have searched the WISE Preliminary Release Database made public in April 2011 for IR images of stars in the VIIth catalogue of Galactic Wolf-Rayet stars (van der Hucht 2001) and found a sample of 31 WR stars with available data ${ }^{1}$. To compare their IR and optical morphologies, we have selected $\mathrm{H} \alpha$ line images of these nebulae in the Super COSMOS Sky Survey (Parker et al. 2005), or STScI Digitized Sky Survey (DSS) ${ }^{2}$ from the Second Palomar Observatory Sky Survey (POSS-II). For a few cases, we have used the [O III] and $\mathrm{H} \alpha$ images presented in Gruendl et al. (2000) that were kindly provided by Y.-H. Chu and R.A. Gruendl. The names and coordinates of the sources in our sample are listed in Table 1 together with the distance, height over the Galactic Plane, and the spectral type and wind terminal velocity of their central stars. Table 1 also identifies the telescope or public database used to acquire the optical images used in this paper. We note that for the specific cases of WR 7 and WR 136

1 The mid-IR WISE images of the nebulae around WR 43abc, WR91, and WR 93 (NGC 3603, RCW 122, and NGC 6357, respectively) were also examined. These stars belong to young stellar clusters, and thus their nebulae are influenced not only by the WR star, but by all stars in the cluster. Their resulting morphologies are complex and difficult to classify. Furthermore, their WISE W3 and W4 band images were saturated at their central regions. Accordingly, we discarded these nebulae from our analysis.

2 https://archive.stsci.edu/cgi-bin/dss_form 

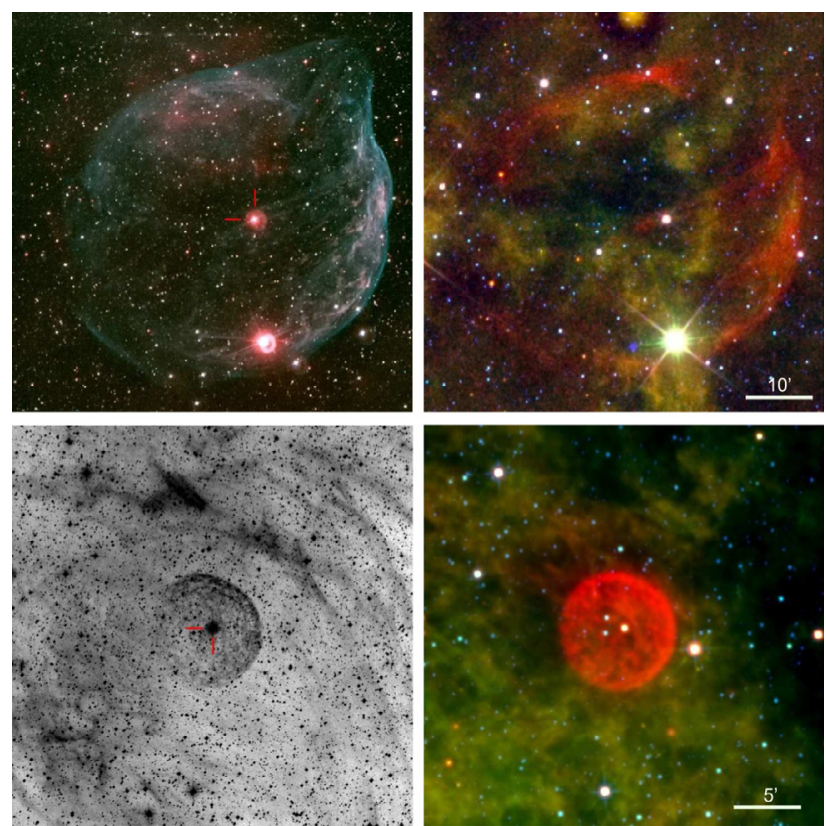

Fig. 1. Examples of WR nebulae with a $\mathcal{B}$-type morphology: WR 6 (top panels) and WR 16 (bottom panels). Left panels: optical morphology while right panels show the WISE W2 (blue), W3 (green), and W4 (red) colour-composite picture. The central WR stars are marked with red lines in the optical images. The colour-composite optical picture of WR 6 was done using $\mathrm{H} \alpha$ (red) and [O III] (blue) narrowband images. North is up, east to the left.

we have also used the existing DSS blue band to complement the morphology seen in the DSS red band ${ }^{3}$.

The WISE images of the WR nebulae in Table 1 were downloaded from the WISE Image search tool at the NASA/IPAC Infrared Science Archive(IRSA). To study the IR morphology of these nebulae, we will use the W2 $\left(\lambda_{\mathrm{c}}=4.6 \mu \mathrm{m}\right)$, W3 $\left(\lambda_{\mathrm{c}}=12 \mu \mathrm{m}\right)$, and $\mathrm{W} 4\left(\lambda_{\mathrm{c}}=22 \mu \mathrm{m}\right)$ bands. The spatial resolutions for the W2, W3, and W4 bands are 6.' 4, 6.'5, and 12.' 0 , respectively, with an astrometric accuracy for bright sources better than $0 . ' 15$.

\subsection{IRS spectra}

To help us interpret the nature of the IR emission of WR nebulae in the WISE W3 and W4 bands, we have searched for Spitzer InfraRed Spectrograph (IRS) spectroscopic observations in the 10-37 $\mu \mathrm{m}$ range of the WR nebulae in our sample listed in Table 1. We found available spectroscopic observations for a sample of $15 \mathrm{WR}$ nebulae. Whereas the detailed analysis of these spectra will be presented in a subsequent paper (Toalá et al., in prep.), here we will focus on the nebulae around WR 7 (NGC 2359), WR 8, and WR 31a, because there are available high-dispersion spectroscopic observations for these nebulae, as well as for the background emission from apertures located at their periphery.

The basic calibrated data (BCD) of the IRS observations of these WR nebulae were downloaded from the Spitzer Heritage Archive. These data are processed with the Spitzer Science Center Pipeline Version S 18.18.0, and include high-resolution spectroscopic observations $(R \sim 600)$ obtained using the ShortHigh (SH, 9.9-19.6 $\mu \mathrm{m})$ and Long-High (LH, 18.7-37.2 $\mu \mathrm{m})$

\footnotetext{
The rest of the WR nebulae do not have their corresponding DSS blue band images.
}
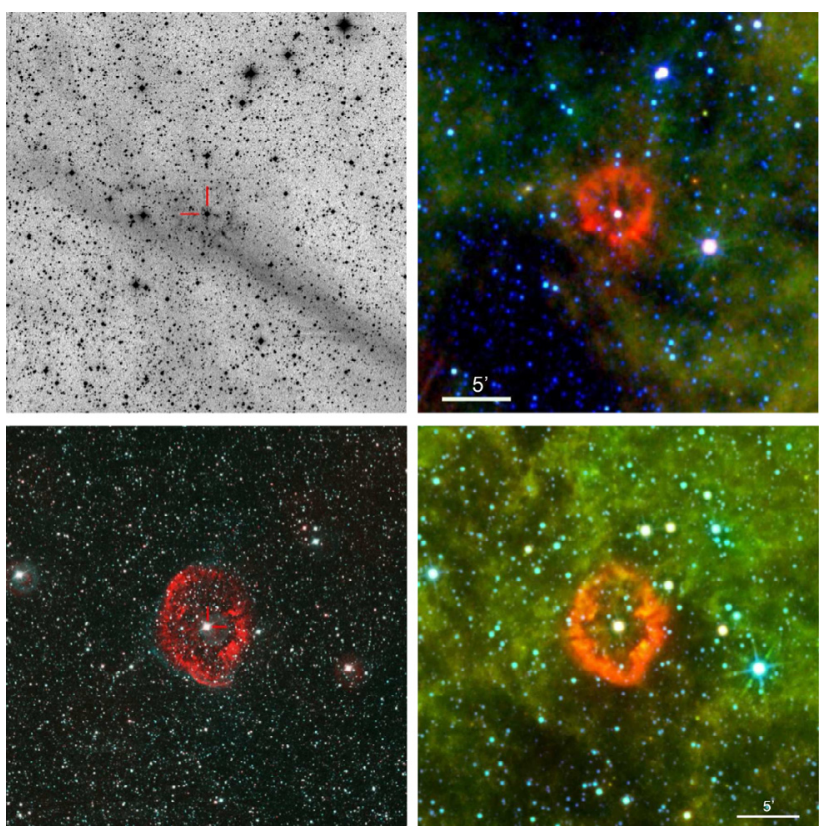

Fig. 2. Same as Fig. 1 but for the cases of WR nebulae with a $C$-Clumpy morphology: WR 8 (top panels) and WR 40 (bottom panels). The colour-composite optical image of WR 40 was done using $\mathrm{H} \alpha$ (red) and [O III] (blue) narrowband images.
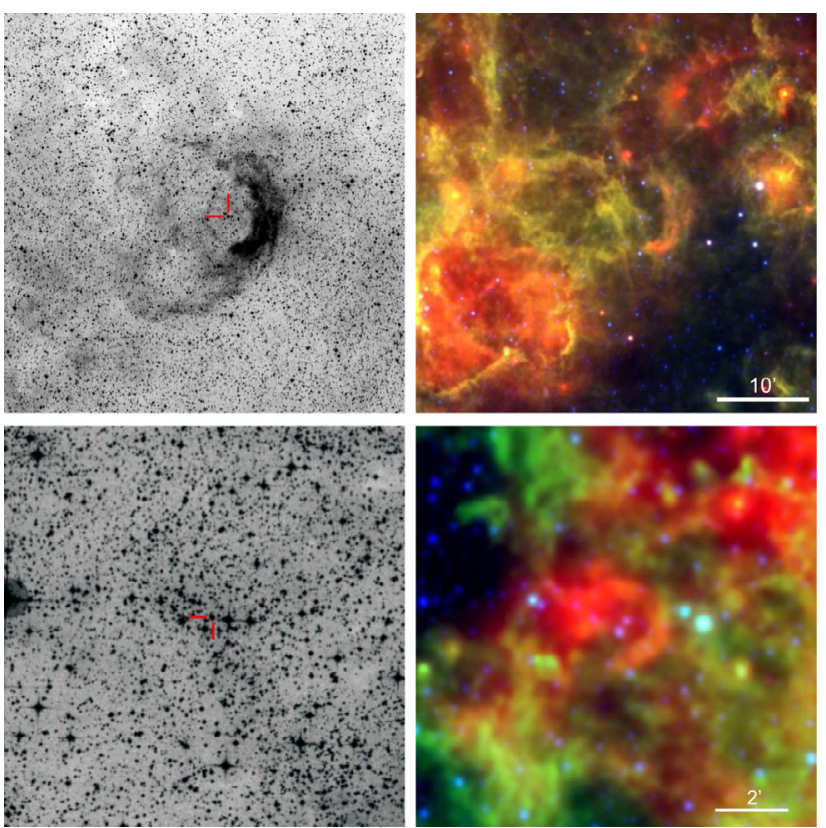

Fig. 3. Same as Fig. 1 but for the cases of WR nebulae with a C-Disrupted morphology: WR 18 (top panels) and WR 35 (bottom panels).

modules. The targets were observed using apertures with sizes of $133^{\prime \prime} 6 \times 44^{\prime \prime} 5$ in the SH module and $22^{\prime \prime} 3 \times 88^{\prime \prime} 9$ in the LH module. All the IRS data were reduced using the CUbe Builder for IRS Spectra Maps (CUBISM). This tool does not only reduce the IRS data, but it can also be used to analyze the IRS data and to extract one-dimensional spectra. The various processing steps followed within CUBISM, including the characterization of noise in the data and the removal of bad pixels, are described by Smith et al. (2007). We note that the Spitzer IRS spectra of WR 8 and WR 31a include the stellar continuum from their 

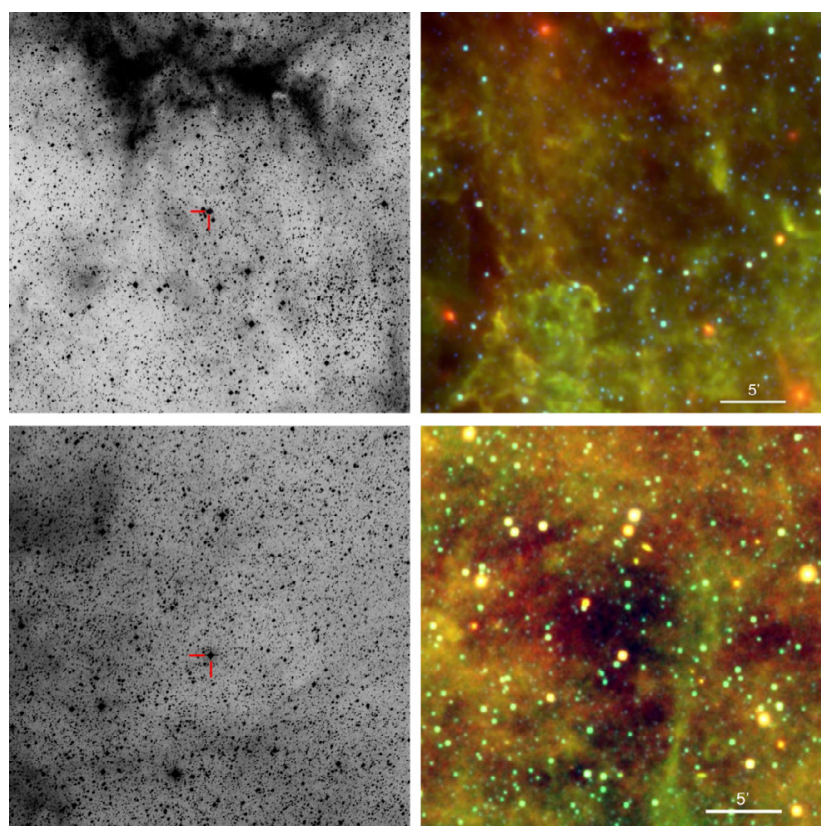

Fig. 4. Same as Fig. 1 but for the cases of WR nebulae with a $\mathcal{M}$ morphology: WR 35b (top panels) and WR 52 (bottom panels).

WR stars, because they were included in the aperture given the small angular size of these nebulae.

\section{Interpreting the IR emission of WR nebulae}

In Figs. 1 to 4 and the figures in Appendix A we present the optical and WISE IR images of the WR nebulae listed in Table 1. The inspection of the colour-composite IR pictures of WR nebulae presented in the figures (see Figs. 1 to 4 ) reveals the prevalence of the emission in the WISE W4 band at $22 \mu \mathrm{m}$ (red in these colour-composite pictures). The emission in this band is mostly coincident with the optical $\mathrm{H} \alpha$ emission from ionized nebular material (e.g. WR 16 in Fig. 1, bottom panels). This is in agreement with Spitzer MIPS $24 \mu \mathrm{m}$ imaging of nebulae around evolved massive stars (e.g. Gvaramadze et al. 2009, 2010a; Wachter et al. 2010; Flagey et al. 2011). On the other hand, the emission in the W3 band (green in the colourcomposite pictures), which is sensitive to cold gas or low ionization material, seems to trace mostly ISM gas along the line of sight (e.g. Fig. 4), with little contribution of emission from the WR nebula in some cases (e.g. S 308 around WR 6, Fig. 1). Some WR nebulae, however, may show bright emission in this band from discrete knots and clumps inside the nebular shell (e.g. NGC 6888, Fig. A.23). Finally, the W2 band (blue in the colour-composite pictures), which is expected to trace the continuum emission from small grains and the background stellar component (see Flagey et al. 2011), shows emission from stars in the background. For the most dusty cases, the nebulae around WR 7, WR 18, WR 22, and WR 23 the W2 band also includes some nebular emission.

The nature of the emission in the WISE W3 and W4 bands has been further investigated using the Spitzer IRS highdispersion background-subtracted spectra of the nebulae around WR 7 (NGC 2359), WR 8, and WR 31a presented in Fig. 5. These spectra have been overplotted by the spectral responses of the WISE W3 (green) and W4 (red) bands. The spectra of these nebulae imply significant contribution to the emission detected in the WISE W3 band by a number of emission lines, including [S IV] $\lambda 10.51 \mu \mathrm{m}, \mathrm{He}$ I $\lambda 10.66 \mu \mathrm{m}, \mathrm{He}$ I $\lambda \lambda 11.31,12.37,16.21 \mu \mathrm{m},[\mathrm{Ar} \mathrm{V}] \lambda 13.10 \mu \mathrm{m}$, and [Ne III] $\lambda 15.55 \mu \mathrm{m}$. In sharp contrast, the WISE W4 band is mostly dominated by continuum emission probably from thermal dust continuum emission, with weak $\mathrm{H} \mathrm{I}$, [Fe II], and [O IV] emission lines. It is interesting to note that the spectrum of WR 7, extracted from a region known to be overabundant in oxygen (Dufour 1989), does not show bright emission from oxygen lines, but a weak [O IV] $\lambda 25.59 \mu \mathrm{m}$ line.

We thus conclude that the emission from WR nebulae in the WISE W3 band is dominated by $\mathrm{H}$ I and forbidden emission lines, mostly [S IV] and [Ne III], with little contribution to the observed emission from dust thermal continuum. Interestingly, the spatial correspondence between broad arc-like patches of emission in the W3 image of S 308 around WR 6 and diminished emission in $\mathrm{H} \alpha$ (Fig. 1) seems to imply absorption of nebular emission by dust on the foreground. As expected, the W3 band traces material in the ISM in most cases. Meanwhile, the emission in the WISE W4 band, tracing mostly the optical nebular shells, corresponds to thermal continuum emission, with very little contribution from $\mathrm{H} \mathrm{I}$, [Fe II], and [O IV] emission lines. These results are not in disagreement with the IRS spectrum of the WR bubble MB 3957, which is dominated by emission lines of iron, sulfur and neon, but also includes an additional contribution of thermal dust emission (Flagey et al. 2011). Otherwise, the Spitzer MIPS $24 \mu \mathrm{m}$ images of planetary nebulae include important contributions from the [O IV] $\lambda 25.89 \mu \mathrm{m}$ and [Ne v] $\lambda 24.3 \mu \mathrm{m}$ emission lines (e.g. Chu et al. 2009), unlike the WISE W4 images of WR nebulae. The spatial resolution of the WISE W4 band images, $\approx 12$ '. 0 , does not allow us to determine whether the dust in WR nebulae is spatially coincident with the ionized material or is found at the leading edge of the optical WR nebulae.

\section{Morphological classification of WR nebulae}

The images of the WR nebulae presented in Figs. 1 to 4 are examples of the variety of morphologies from complete shells, arcs (incomplete shells), clumps, and filaments to diffuse, featureless emission. A close inspection of all WR nebulae in our sample and the comparison between optical and IR morphologies has led us to classify them into three broad morphological types:

\section{- $\mathcal{B}$ - WR bubble.}

These nebulae present a thin shell or bubble both in optical and IR, mostly in the W4 band. Examples of these nebulae are those around WR 6 (S 308) and WR 16 (see Fig. 1).

- C - Clumpy/disrupted WR bubbles.

These nebulae present clumpy $\mathrm{H} \alpha$ and IR images and/or incomplete arcs or shells. The W4 images reveal for a significant number of cases that the optical and IR clumps are spatially coincident with bow shock-like features. Archetypes of these WR nebulae are those around WR 8 and WR 18, WR 35 and WR 40 (see Figs. 2 and 3).

- $M$ - Mixed WR nebulae.

These nebulae do not present a clear correspondence between the optical and IR images. Indeed, they do not show a clearly defined IR morphology, with a noticeable lack of emission in the WISE W4 band. Examples of these nebulae are those around WR 35b and WR 52 (see Fig. 4).

Our classification of the WR nebulae is listed in the last column of Table 1. We note that sometimes the assignation of a morphological type is difficult given the disparity between the optical 


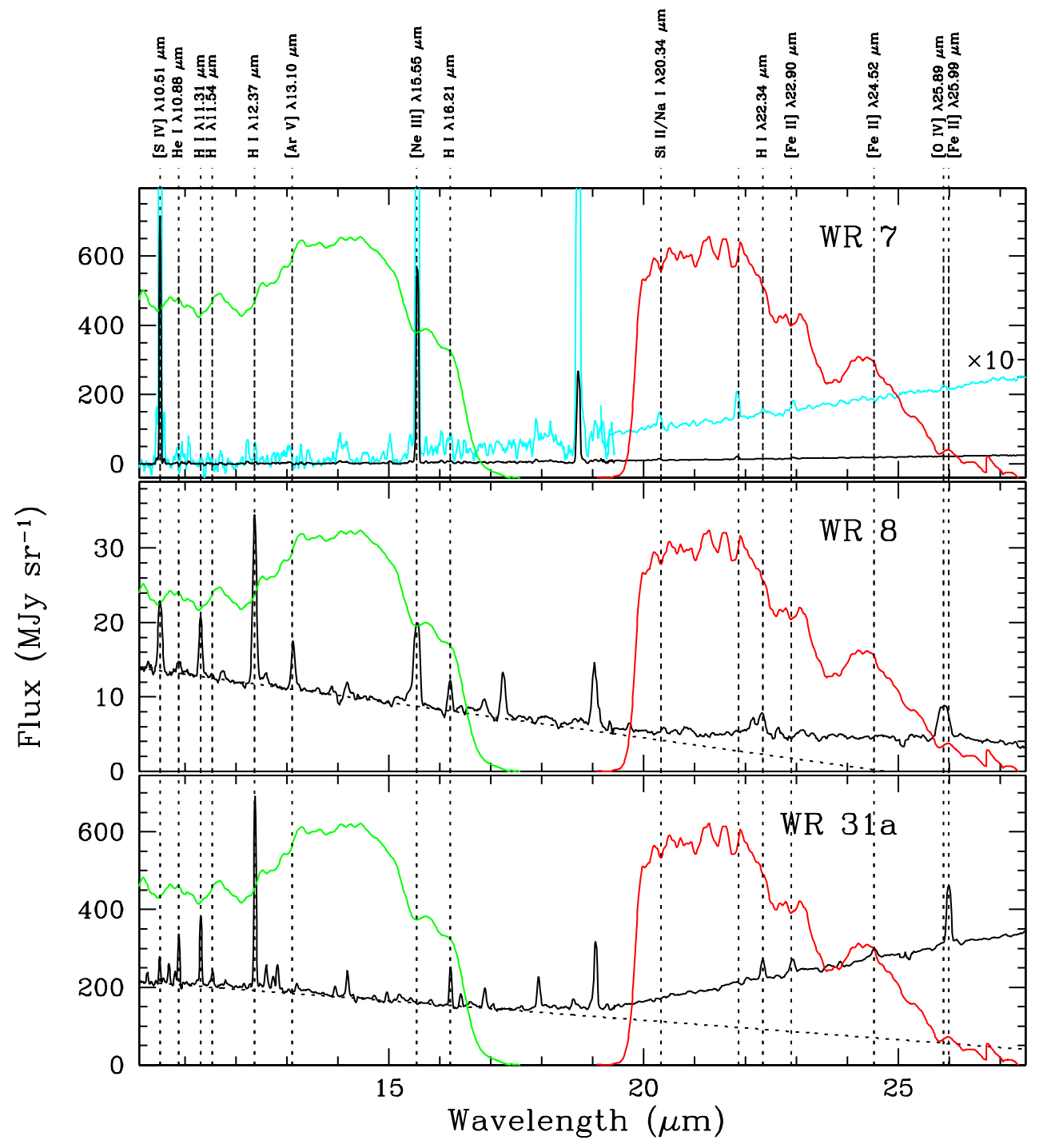

Fig. 5. Spitzer IRS SH and LH spectra of the nebulae around WR 7, WR 8, and WR 31a. The spectral responses of the W3 (green) and W4 (red) WISE bands normalized to arbitrary values are overplotted on the spectra. The nebular spectrum of WR 7 corresponds to the patch of bright red emission (the WISE W4 band) towards the northwest shown in Fig. A.1. This spectrum has been plotted at two different intensity levels to highlight the low level emission. The contribution of the stellar emission of WR 8 and WR 31a to the nebular spectra can be roughly reproduced by a blackbody model (dashed line). and IR images or because of the ambiguous association between the star and the nebula. For instance, the nebula around WR 116 (Fig. A.17) has been assigned a morphological type $C$ on the basis of its optical image, but a morphological type $\mathcal{M}$ cannot be completely excluded by its IR counterpart.

\subsection{Notes on individual objects}

\subsubsection{WR 35 and its obscured shell}

We report the previously unknown partial shell around WR 35. This shell, clearly detected in the WISE W4 band (Fig. 3, bottom panels), is $\sim 2$ ! $0 \times 1$ '.5 in size and opens towards the southeast of WR 35. In contrast, the $\mathrm{H} \alpha$ image from the Super COSMOS Sky Survey only detects emission from an ionized cloud southwest of WR 35, which does not coincide spatially with the IR shell. A similar case of detection of an obscured shell has been claimed by Wachter et al. (2011) for WR 8, although an optical nebulosity was previously reported by Stock \& Barlow (2010). The high frequency of discovery of obscured shells around massive stars in Spitzer MIPS $24 \mu \mathrm{m}$ images has led to the suggestion that such shells may be ubiquitous among these stars (e.g. Gvaramadze et al. 2010a; Wachter et al. 2010).

\subsubsection{A bipolar nebula around WR 85}

The nebula RCW 118 around WR 85 has been described as a bipolar nebula based on $\mathrm{H} \alpha$ images obtained by Marston et al. (1994a,b). This bipolar structure is hinted in the WISE W4 image by the red patches to the northeast and southwest of WR 85 (Fig. A.11, right). This IR image is certainly reminiscent of the optical image of the nebula NGC 6164-5 around the O6 star HD 148937 (Bruhweiler et al. 1981; Dufour et al. 1988).

\subsection{Comparison with previous morphological classifications}

Previous imaging studies of WR nebulae have disclosed a rich morphological variety among them (e.g. Chu et al. 1983; Gruendl et al. 2000; Stock \& Barlow 2010). The most complete classification scheme of WR nebulae is that originally introduced by Chu (1981) and more recently described by Chu (2003). This classification scheme relies on narrowband optical images, kinematics, and information on the chemical abundances to define the presence of bubbles and their expansion rate and kinematical age. Meanwhile, our classification scheme of WR nebulae is supported by the comparison between narrowband optical (mostly $\mathrm{H} \alpha$ ) and mid-IR WISE images, the latter being very useful for unveiling obscured shells and disentangling 
Table 2. Comparison of WR nebula morphology classification.

\begin{tabular}{cccl}
\hline \hline WR & This paper & Previous works & References \\
\hline 6 & $\mathcal{B}$ & $W / E$ & $1,2,3,4$ \\
7 & $\mathcal{B}$ & $W$ & $1,3,4,5$ \\
8 & $C$ & $E$ & 4 \\
16 & $\mathcal{B}$ & $W$ & 4,7 \\
18 & $\mathcal{M}$ & $W$ & $1,3,4,8$ \\
22 & $\mathcal{M}$ & $W$ & 3,6 \\
23 & $\mathcal{B}$ & $W$ & $1,3,7$ \\
38 & $\mathcal{M}$ & Ring? & 7 \\
40 & $\mathcal{M}$ & $E$ & $1,3,4,8$ \\
52 & $\mathcal{M}$ & $R_{\mathrm{s}}$ & $1,3,9$ \\
54 & $\mathcal{M}$ & Ring? & 6 \\
55 & $\mathcal{C}$ & $R_{\mathrm{a}}$ & $1,3,9$ \\
68 & $\mathcal{M}$ & $\mathrm{Ring}$ & 6 \\
75 & $\mathcal{B}$ & $W / E$ & $1,3,4,8$ \\
85 & $\mathcal{C}$ & $R_{\mathrm{s}}$ & $1,3,9$ \\
86 & $\mathcal{M}$ & $\ldots$ & 6 \\
94 & $\mathcal{M}$ & $\ldots$ & 7 \\
95 & $\mathcal{C}$ & $\ldots$ & 6 \\
101 & $\mathcal{B}$ & Ring? & 7 \\
102 & $\mathcal{B}$ & $W / E$ & 4,7 \\
124 & $\mathcal{B}$ & $E$ & $1,3,4,10$ \\
128 & $\mathcal{B}$ & $W / R_{\mathrm{s}}$ & 3,11 \\
131 & $\mathcal{C}$ & $\mathrm{H}$ II & $1,3,10$ \\
134 & $\mathcal{B}$ & $W$ & $1,3,12$ \\
136 & $\mathcal{B}$ & $W / E$ & $1,3,4,12$ \\
\hline & & &
\end{tabular}

References. (1) Chu (1981); (2) Chu et al. (1982); (3) Chu et al. (1983); (4) Stock \& Barlow (2010); (5) Treffers \& Chu (1982a); (6) Marston et al. (1994a); (7) Marston et al. (1994b); (8) Chu (1982); (9) Chu \& Treffers (1981b); (10) Chu \& Treffers (1981a); (11) Gruendl et al. (2000); (12) Treffers \& Chu (1982b).

the nebular emission from background diffuse and ISM emission as discussed in Sect. 3.

The comparison between these two morphological classifications of WR nebulae in Table 2 reveals a good agreement. The agreement is excellent for the wind-blown bubbles (our $\mathcal{B}$ nebulae and the $W$ class), because they are the easieast to spot in direct images or kinematical data. Indeed, all our $\mathcal{B}$ nebulae are classified as $W$, and only four $W$ objects are classified as $C$ nebulae because of their mid-IR morphology.

This consistency is confirmed by the comparison between optical images in the $\mathrm{H} \alpha$ and [O III] emission lines, which has been shown by Gruendl et al. (2000) to be very helpful in revealing the expansion of shock fronts outside the main nebular shell. For example, in the case of RCW 104 around WR 75 (Fig. A.10), the $\mathrm{H} \alpha$ line image does not show any morphological characteristic of a round shell, thus implying a classification as a $C$-type nebula. Instead, the [O III] morphology implies a $\mathcal{B}$-type. On the contrary, the nebula M 1-67 around WR 124 shows no evidence of an [O III] front (Fernández-Martín et al. 2013, and references therein), but its kinematics reveals an expanding shell consisting of numerous condensations (Solf \& Carsenty 1982). While these findings may raise doubts for the classification of WR nebulae for which [O III] images and kinematics are not available, we note that in all these nebulae the outer expanding shell is noticeably much fainter than the shells detected in our $\mathcal{B}$-type nebulae. This points to an intrinsic difference between the two morphological types.

Of the $\mathcal{M}$ nebulae, only two have previous definite morphology (Chu 1991). The nebula around WR 22 is described by Chu (1991) as a possible wind-blown bubble, although no evidence for such a bubble is revealed in WISE images (Fig. A.2). Similarly, the nebula around WR 52 is categorized as an $R_{\mathrm{S}}$ nebula on the basis of kinematical data, but the nebula is rather inconspicuous in the IR (Fig. 4, bottom panels).

\subsection{Interpreting the morphology of WR nebulae}

The main morphological characteristics of WR nebulae result from the interactions of the WR wind and the intense UV radiation with the slow wind ejected previously by the progenitor. The most basic evolutionary path of a WR nebula, as reproduced by multiple simulations (e.g. García-Segura \& Mac Low 1995; García-Segura et al. 1996a,b; Freyer et al. 2003; van Marle et al. 2005; Freyer et al. 2006; Arthur 2007a,b; van Marle et al. 2007; Dwarkadas 2007; Toalá \& Arthur 2011), implies a massive star ejecting a significant fraction of its mass during a RSG or LBV phase through a spherical, slow $\left(10-100 \mathrm{~km} \mathrm{~s}^{-1}\right)$, and dense wind. In the final stage of stellar evolution, during the WR phase, this CSM will be swept up by the WR fast stellar wind $\left(v_{\infty} \gtrsim 10^{3} \mathrm{~km} \mathrm{~s}^{-1}\right)$, creating at early times a WR bubble $(\mathcal{B}$ type nebulae). Later on, the WR bubble will be disrupted due to Rayleigh-Taylor and thin shell instabilities ( $C$-type nebulae) and the resulting filaments and clumps will later mix with the ISM ( $\mathcal{M}$-type nebulae).

Additional processes may alter this general scheme. For instance, the proper motion of the star or a density gradient in the ISM may compress and brighten the nebula as it interacts with its surroundings, resulting in incomplete shells and arcs. Stellar rotation can also introduce changes in the stellar evolution (e.g. Meynet \& Maeder 2003; Ekström et al. 2012) and produce, for instance, anisotropic stellar winds. In the case of non-spherical (e.g. bipolar or clumpy) ejections of material seen in some LBV nebulae (e.g. MN13 and MN79; Humphreys 2010; Gvaramadze et al. 2010a), the wind-wind interaction will give place to the immediate formation of clumps or filaments, forming directly a $C$-type nebulae with no B-type nebula in between. The largescale structure of the ISM may also alter the morphology of WR nebulae.

Since WR nebulae can form through different morphological sequences, we should not expect a tight correlation between nebular morphology and the star evolutionary stage implied by its WR spectral type (e.g. Moffat 1995; Maeder 1997). Certainly, $\mathcal{M}$-type nebulae can be expected preferentially around evolved WR stars, whereas the central stars of $\mathcal{B}$ - and $\mathcal{C}$-type nebulae can be expected to have late spectral types (Chu 1981; Chu et al. 1983). This correspondence is further complicated by the time-lapse spent by a WR star on the different WR subphases which depends strongly on different factors (initial mass, rotation, initial metallicity; e.g. Maeder 1991; Meynet \& Maeder 2005; Georgy et al. 2012). A star may even miss a WR subphase; for example, the stellar evolution models at solar metallicity of Meynet \& Maeder (2003) predict that a rotating $25 M_{\odot}$ star will not enter the WNE and WC stages. Therefore, for those particular models, any type of WR nebulae can be found around WNL stars because the long duration of this phase or even the lack of the WNE stage. On the other hand, for the case of non-rotating models, which predict comparable durations of the WNL and WNE phases, we may expect $\mathcal{B}$-type nebulae around these two types of stars.

To better quantify these relationships, in Fig. 6 we show the distributions of nebular morphology and stellar spectral type among the WR nebulae in our sample. Five out of the nine $\mathcal{B}$ nebulae have WNE stars and two others have WNL spectral types, whereas only two are early WC stars. The WNL stars 


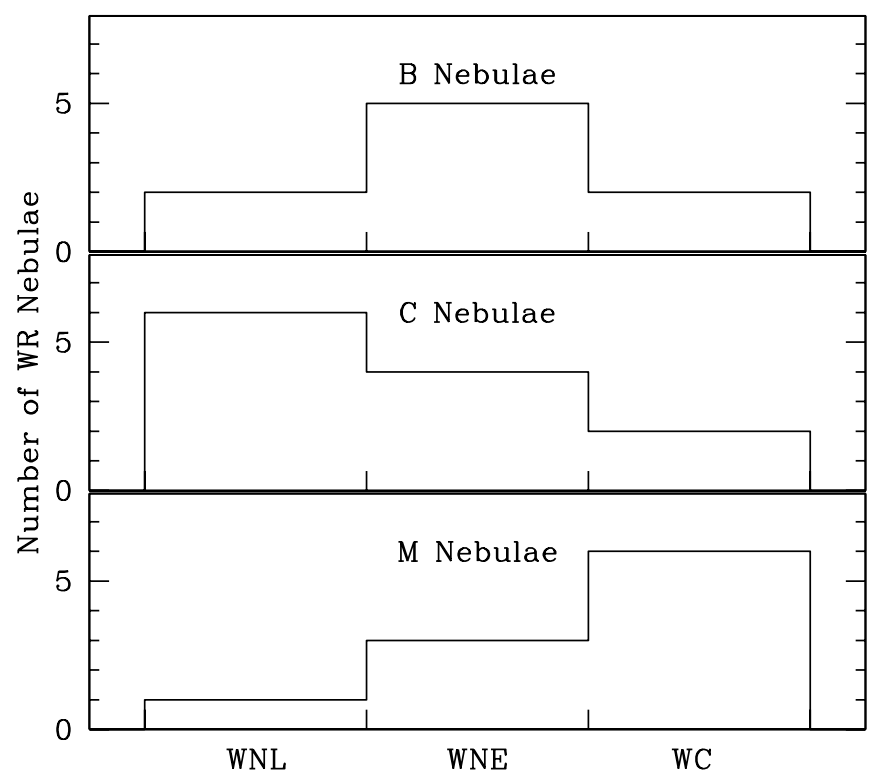

Fig. 6. Distribution of the different morphological types of WR nebulae depending on the spectral types of their WR central stars.

WR 16 (WN8) and WR 31a (WN11) are surrounded by smallsized bubbles (2.8 and $1.2 \mathrm{pc}$ in radius, respectively), and the WC WR 23 (WC6) and WR 102 (WO2) stars by large bubbles (16 and $4 \mathrm{pc}$ in radius, respectively). Nebulae with $C$-type morphology harbor late WN7-WN8 stars (6 out of 12) or WNE stars (4 out of 12), with only two WC stars (WR 95 and WR 101). On the contrary, $\mathcal{M}$ nebulae have a significant fraction of early WC stars (6 out of 10) and a very small number of WNL stars (1 out of 10). This confirms previous suggestions that WR bubbles are associated with late WN stars and $C$-type to WC stars. Most $C$ nebulae had been previously classified either as $W$ or $E$ nebulae, but we notice some contamination of $R_{\mathrm{a}}$ and $R_{\mathrm{S}}$ nebulae, and even objects of uncertain morphology. This indicates that $C$ nebulae have a variety of kinematical and chemical properties. This is also the case for their WR central stars which can be linked to different evolutionary paths leading to a $C$-nebula morphology.

\section{Comparison with previous mid-IR studies}

The extinction by dust in the Galactic Plane makes it difficult to search for WR nebulae at optical wavelengths. The detection of the mid-IR emission (thermal dust, molecules, PAHs, etc.) of shells around evolved stars may provide an alternative method for the direct identification of these nebulae. Mid-IR observations have indeed allowed the detection of young PNe (e.g. Morris et al. 2006; Fesen \& Milisavljevic 2010) and LBV nebulae (e.g. Gvaramadze et al. 2010b; Wachter et al. 2010). Not unexpectedly, Spitzer MIPS $24 \mu \mathrm{m}$ data have been used to discover new WR stars by the direct detection of mid-IR emission from its circumstellar nebula (e.g. WR 121b, Gvaramadze et al. 2010c). Two outstanding examples are the identification of the interacting nebulae around the WN9h type stars, WR 120bb and WR 120bc, by Burgemeister et al. (2013) and the identification of a WR nebula in the Large Magellanic Cloud reported by Gvaramadze et al. (2014). The direct identification of circumstellar nebulae around WR stars in the mid-IR $24 \mu \mathrm{m}$ band requires a mandatory spectroscopic characterization of the nebular emission, but it is important to emphasize the simplicity and potential of the mid-IR method.
In this paper, we have taken advantage of the similarities between the spectral responses of the Spitzer MIPS $24 \mu \mathrm{m}$, and WISE W4 $22 \mu \mathrm{m}$ bands to investigate the circumstellar medium around a significant sample of known Galactic WR stars. By identifying the mid-IR counterparts of these WR nebulae, we have assessed their true extent, disentangling the optical emission of the circumstellar shells from that of the ISM along the line of sight. New mid-IR shells (e.g. WR 35, Fig. 3, bottom panels) have also been discovered. It is worth mentioning here the notable case of NGC 3199, the nebula around WR 18 (see Fig. 3, top panels). Stock et al. (2011) derived the chemical abundances for a bright $\mathrm{H} \alpha$ nebular clump and concluded they were consistent with those of the Galactic H II region M 17 on which NGC 3199 is projected. Accordingly, they proposed that NGC 3199 consists mainly of swept-up ISM material. A careful registration of the location of the $\sim 15^{\prime \prime}$ VLT/UVES slit used by Stock et al. (2011) on the WISE images shows that this small slit does not include the emission detected in the W4 band (i.e. nebular material), but it is located on a spot of bright emission in the W3 band (i.e. ISM material). These findings cast doubt on the ISM abundances implied by those authors for NGC 3199 and instead support those reported by Marston (2001).

\section{Summary and conclusions}

We have examined WISE IR images of a sample of 31 WR stars to study their nebular morphologies in mid-IR bands. We have then compared the emission in the WISE images with optical $\mathrm{H} \alpha$ and (in some cases) [O III] emission line images. The variety of morphologies of WR nebulae can be classified as bubble $\mathcal{B}$-type nebulae, clumpy/disrupted $C$-type nebulae, and mixed $\mathcal{M}$-type nebulae.

We have used Spitzer IRS spectra of some WR nebulae to investigate the nature of the IR emission of WR nebulae in the different WISE bands (Toalá et al., in prep.). According to previous IR studies of evolved massive stars, we find that the emission in the WISE W4 band of $\mathcal{B}$ and $C$-type nebulae most likely traces dust associated to the WR nebula. On the other hand, the WISE W3 band mainly traces the emission of material along the line of sight of the nebula, which results in diminished emission at optical wavelengths. We emphasize that the acquisition of mid-IR images of WR nebulae, especially for wavelengths above $20 \mu \mathrm{m}$, is very useful for disentangling the emission from the WR nebula from that of the ISM. This provides a less demanding observational approach for the identification and study of WR nebulae than those based on optical images and spectra. The discovery of an obscured shell around WR $35 \mathrm{~m}$, which is only detected at $22 \mu \mathrm{m}$ in the W4 band WISE images, or the ability to distinguish the nebular material of NGC 3199 around WR 18 from that of the Galactic H II region M 17 clearly illustrate the advantages of mid-IR observations of WR nebulae.

We find a loose correlation between nebular morphology and stellar spectral type, also claimed in the past, but we note that this correlation is complicated by the different evolutionary sequences of WR nebulae and the dependence of the stellar evolution in these phases with initial mass and composition, and stellar rotation.

Acknowledgements. We would like to thank the referee, Margaret Meixner, for helpful comments. J.A.T. acknowledges CSIC JAE-PREDOC (Spain) student grant 2011-00189. J.A.T. and M.A.G. are partially funded by grants AYA 2008-01934 and AYA 2011-29754-C03-02 of the Spanish MICINN (Ministerio de Ciencia e Innovación) and MEC (Ministerio de Economía y Competitividad) including FEDER funds. GR-L acknowledges support from 
CONACyT (grant 177864) and PROMEP (Mexico). We are grateful to Y.-H. $\mathrm{Chu}$ and R.A. Gruendl for providing us with the [O III] and $\mathrm{H} \alpha$ optical images of S 308, RCW 58, RCW 104, WR 128, and WR 134. This paper is based on observations from the Wide-field Infrared Survey Explorer, which is a joint project of the University of California, Los Angeles, and the Jet Propulsion Laboratory/California Institute of Technology, founded by the National Aeronautics and Space Administration. The Digitized Sky Surveys were produced at the Space Telescope Science Institute under the U.S. Goverment grant NAG W-2166. The Second Palomar Observatory Sky Survey (POSS-II) was made by the California Institute of Techonology with funds from the National Science Foundation, the National Geographic Society, the Sloan Foundation, the Samuel Oschin Foundation, and the Eastman Kodak Corporation.

\section{References}

Arnal, E. M., \& Cappa, C. E. 1996, MNRAS, 279, 788

Arnal, E. M., Cappa, C. E., Rizzo, J. R., \& Cichowolski, S. 1999, AJ, 118, 1798

Arthur, S. J. 2007a, Rev. Mex. Astron. Astrofis. Conf. Ser., 30, 64

Arthur, S. J. 2007b, Diffuse Matter from Star Forming Regions to Active Galaxies, 183

Bochkarev, N. G. 1988, Nature, 332, 518

Bruhweiler, F. C., Gull, T. R., Henize, K. G., \& Cannon, R. D. 1981, ApJ, 251, 126

Burgemeiste, S., Gvaramadze, V. V., Stringfellow, G. S., et al. 2013, MNRAS, 429, 3305

Cappa, C. E., Goss, W. M., \& Pineault, S. 2002, AJ, 123, 3348

Cappa, C. E., Vasquez, J., Arnal, E. M., Cichowolski, S., \& Pineault, S. 2008,

Mass Loss from Stars and the Evolution of Stellar Clusters, 388, 151

Cappa, C. E., Rubio, M., Martín, M. C., \& Romero, G. A. 2009, A\&A, 508, 759

Chu, Y.-H. 1981, ApJ, 249, 195

Chu, Y.-H. 1982, ApJ, 254, 578

Chu, Y.-H. 1991, Wolf-Rayet Stars and Interrelations with Other Massive Stars in Galaxies, 143, 349

Chu, Y.-H. 2003, A Massive Star Odyssey: From Main Sequence to Supernova, 212,585

Chu, Y.-H., \& Treffers, R. R. 1981a, ApJ, 249, 586

Chu, Y.-H., \& Treffers, R. R. 1981b, ApJ, 250, 615

Chu, Y.-H., Gull, T. R., Treffers, R. R., \& Kwitter, K. B. 1982, ApJ, 254, 562

Chu, Y.-H., Treffers, R. R., \& Kwitter, K. B. 1983, ApJS, 53, 937

Chu, Y.-H., Guerrero, M. A., Gruendl, R. A., García-Segura, G., \& Wendker, H. J. 2003, ApJ, 599, 1189

Chu, Y.-H., Gruendl, R. A., Guerrero, M. A., et al. 2009, AJ, 138, 691

Dufour, R. J. 1989, Rev. Mex. Astron. Astrofis., 18, 87

Dufour, R. J., Parker, R. A. R., \& Henize, K. G. 1988, ApJ, 327, 859

Dwarkadas, V. V. 2007, ApJ, 667, 226

Ekström, S., Georgy, C., Eggenberger, P., et al. 2012, A\&A, 537, A146

Fernández-Martín, A., Martín-Gordón, D., Vílchez, J. M., et al. 2012, A\&A, 541, A119

Fernández-Martín, A., Vílchez, J. M., Pérez-Montero, E., et al. 2013, A\&A, 554, A104

Fesen, R. A., \& Milisavljevic, D. 2010, AJ, 139, 2595

Flagey, N., Noriega-Crespo, A., Billot, N., \& Carey, S. J. 2011, ApJ, 741, 4

Freyer, T., Hensler, G., \& Yorke, H. W. 2003, ApJ, 594, 888

Freyer, T., Hensler, G., \& Yorke, H. W. 2006, ApJ, 638, 262
García-Segura, G., \& Mac Low, M.-M. 1995, ApJ, 455, 160

García-Segura, G., Mac Low, M.-M., \& Langer, N. 1996a, A\&A, 305, 229

García-Segura, G., Langer, N., \& Mac Low, M.-M. 1996b, A\&A, 316, 13

Georgy, C., Ekström, S., Meynet, G., et al. 2012, A\&A, 542, A29

Gruendl, R. A., Chu, Y.-H., Dunne, B. C., \& Points, S. D. 2000, AJ, 120, 2670

Gvaramadze, V. V., Fabrika, S., Hamann, W.-R., et al. 2009, MNRAS, 400, 524

Gvaramadze, V. V., Kniazev, A. Y., \& Fabrika, S. 2010a, MNRAS, 405, 1047

Gvaramadze, V. V., Kniazev, A. Y., Fabrika, S., et al. 2010b, MNRAS, 405, 520

Gvaramadze, V. V., Kniazev, A. Y., Hamann, W.-R., et al. 2010c, MNRAS, 403, 760

Gvaramadze, V. V., Chené, A.-N., Kniazev, A. Y., et al. 2014, MNRAS, 442, 929

Hamann, W.-R., Gräfener, G., \& Liermann, A. 2006, A\&A, 457, 1015

Humphreys, R. M. 2010, Hot and Cool: Bridging Gaps in Massive Star Evolution, 425, 247

Maeder, A. 1991, A\&A, 242, 93

Maeder, A. 1997, IAU Symp., 189, 313

Marston, A. P. 2001, ApJ, 563, 875

Marston, A. P., Chu, Y.-H., \& García-Segura, G. 1994a, ApJS, 93, 229

Marston, A. P., Yocum, D. R., García-Segura, G., \& Chu, Y.-H. 1994b, ApJS, 95, 151

Mauerhan, J. C., Wachter, S., Morris, P. W., Van Dyk, S. D., \& Hoard, D. W. 2010, ApJ, 724, L78

Meynet, G., \& Maeder, A. 2003, A\&A, 404, 975

Meynet, G., \& Maeder, A. 2005, A\&A, 429, 581

Moffat, A. F. J. 1995, Wolf-Rayet Stars: Binaries; Colliding Winds; Evolution, 163,213

Morris, P. W., Stolovy, S., Wachter, S., et al. 2006, ApJ, 640, L179

Parker, Q. A., Phillipps, S., Pierce, M. J., et al. 2005, MNRAS, 362, 689

Sander, A., Hamann, W.-R., \& Todt, H. 2012, A\&A, 540, A144

Smith, J. D. T., Armus, L., Dale, D. A., et al. 2007, PASP, 119, 1133

Solf, J., \& Carsenty, U. 1982, A\&A, 116, 54

Stock, D. J., \& Barlow, M. J. 2010, MNRAS, 409, 1429

Stock, D. J., Barlow, M. J., \& Wesson, R. 2011, MNRAS, 418, 2532

Stringfellow, G. S., Gvaramadze, V. V., Beletsky, Y., \& Kniazev, A. Y. 2012, Proc. Scientific Meeting in Honor of Anthony F. J. Moffat, Internaional Astronomical Union Symp., ASP Conf. Ser., 465, 514

Toalá, J. A., \& Arthur, S. J. 2011, ApJ, 737, 100

Toalá, J. A., Guerrero, M. A., Chu, Y.-H., et al. 2012, ApJ, 755, 77

Toalá, J. A., Guerrero, M. A., Chu, Y.-H., \& Gruendl, R. A. 2015, MNRAS, 446, 1083

Treffers, R. R., \& Chu, Y.-H. 1982b, ApJ, 254, 569

Treffers, R. R., \& Chu, Y.-H. 1982a, ApJ, 254, 132

van Buren, D., \& McCray, R. 1988, ApJ, 329, L93

van der Hucht, K. A. 2001, New A Rev., 45, 135

van Marle, A. J., Langer, N., \& García-Segura, G. 2005, A\&A, 444, 837

van Marle, A. J., Langer, N., \& García-Segura, G. 2007, A\&A, 469, 941

Wachter, S., Mauerhan, J. C., Van Dyk, S. D., et al. 2010, AJ, 139, 2330

Wachter, S., Cohen, M., \& Leisawitz, D. 2011, BAAS, 43, \#333.10

Wrigge, M. 1999, A\&A, 343, 599

Wrigge, M., \& Wendker, H. J. 2002, A\&A, 391, 287

Wrigge, M., Wendker, H. J., \& Wisotzki, L. 1994, A\&A, 286, 219

Wrigge, M., Chu, Y.-H., Magnier, E. A., \& Wendker, H. J. 2005, ApJ, 633, 248

Wright, E. L., Eisenhardt, P. R. M., Mainzer, A. K., et al. 2010, AJ, 140, 1868

Zhekov, S. A., \& Park, S. 2011, ApJ, 728, 135 
J. A. Toalá et al.: WISE morphological study of WR nebulae

\section{Appendix A: Additional figures}

In this appendix we collect the figures of the rest of the WR nebulae studied in this paper not presented in the main text. The nebulae are those around the WR stars WR 7, WR 22, WR 23, WR 30, WR 31a, WR 38, WR 54, WR 55, WR 68, WR 75, WR 85, WR 86, WR 94, WR 95, WR 101, WR 102, WR 113, WR 116, WR 124, WR 128, WR 131, WR 134, and WR 136.
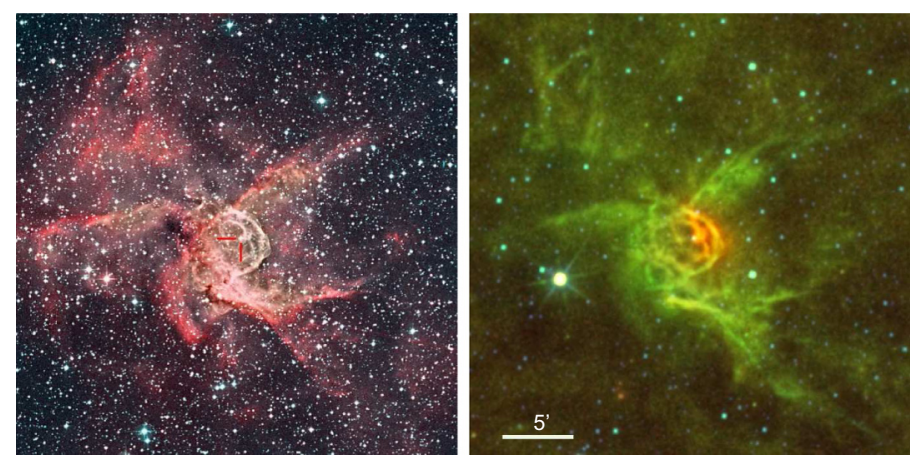

Fig. A.1. Optical (left) and mid-IR WISE (right) images of WR 7 (NGC 2359). See Table 1 for details of the optical image. The central WR star is marked with red lines in the left panel. North is up, east to the left.
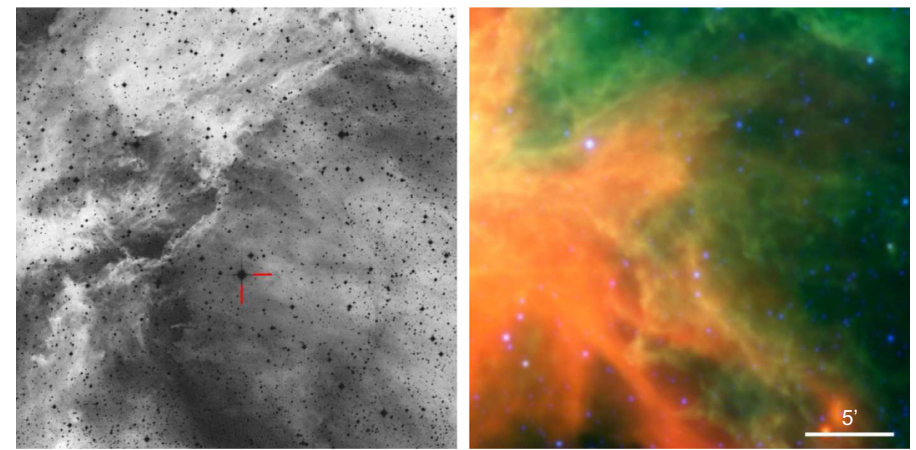

Fig. A.2. Same as Fig. A.1 for WR 22.
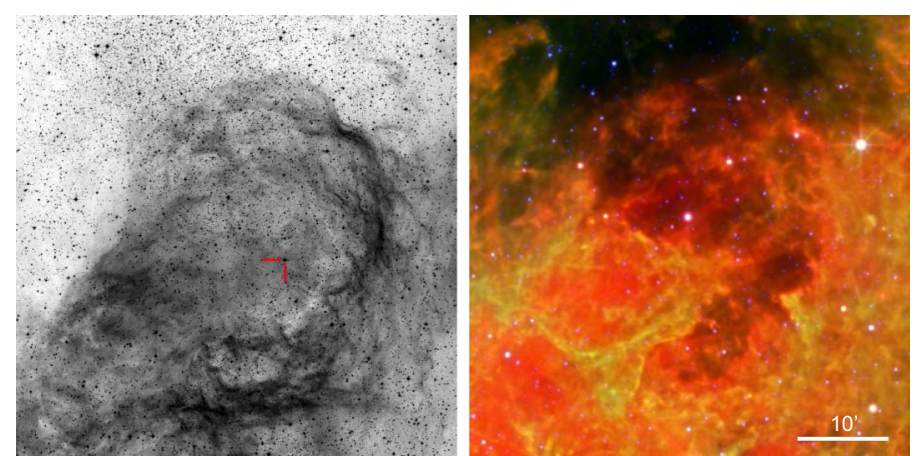

Fig. A.3. Same as Fig. A.1 for WR 23.
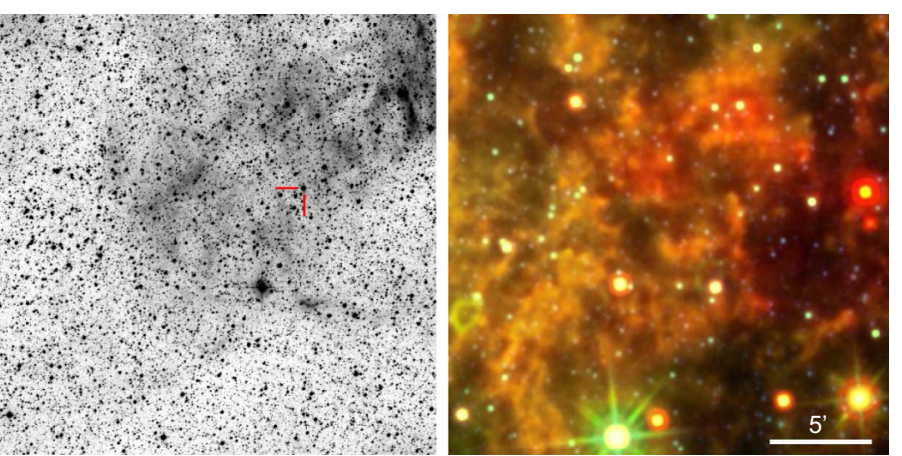

Fig. A.4. Same as Fig. A.1 for WR 30 (Anon).

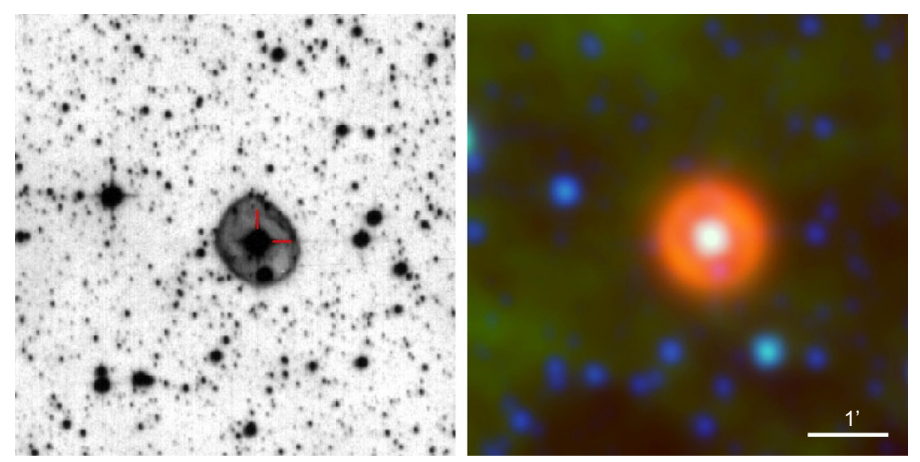

Fig. A.5. Same as Fig. A.1 for WR 31a.
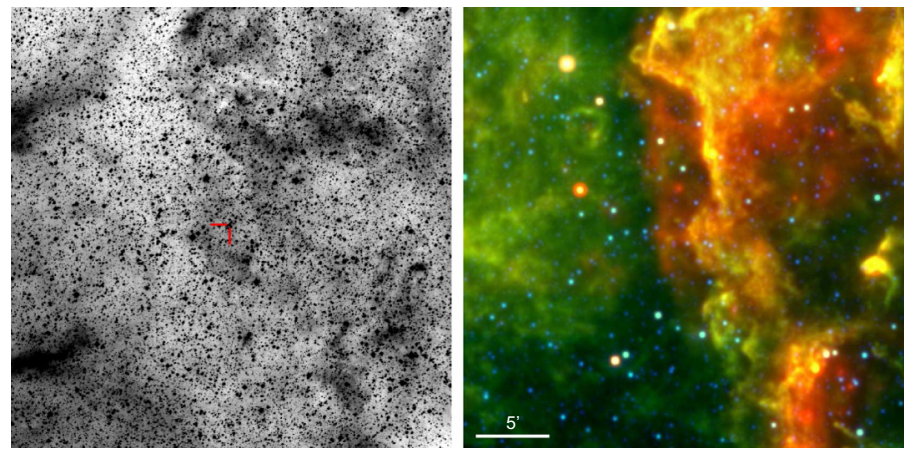

Fig. A.6. Same as Fig. A.1 for WR 38.
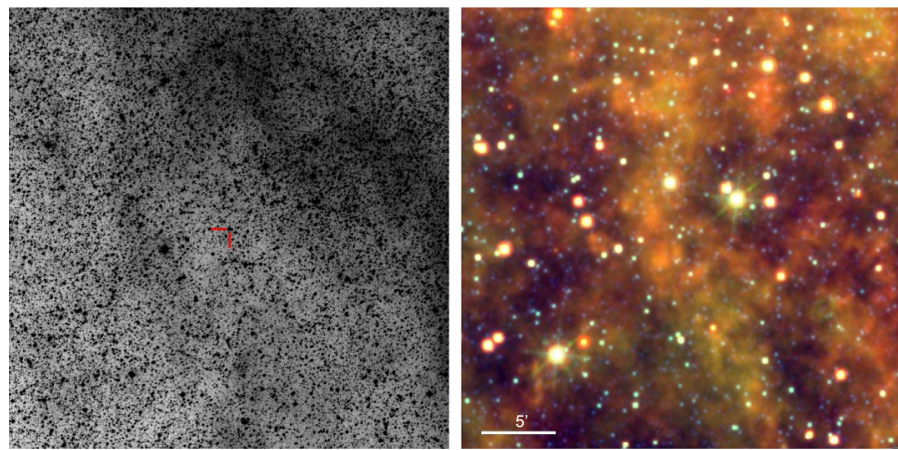

Fig. A.7. Same as Fig. A.1 for WR 54. 

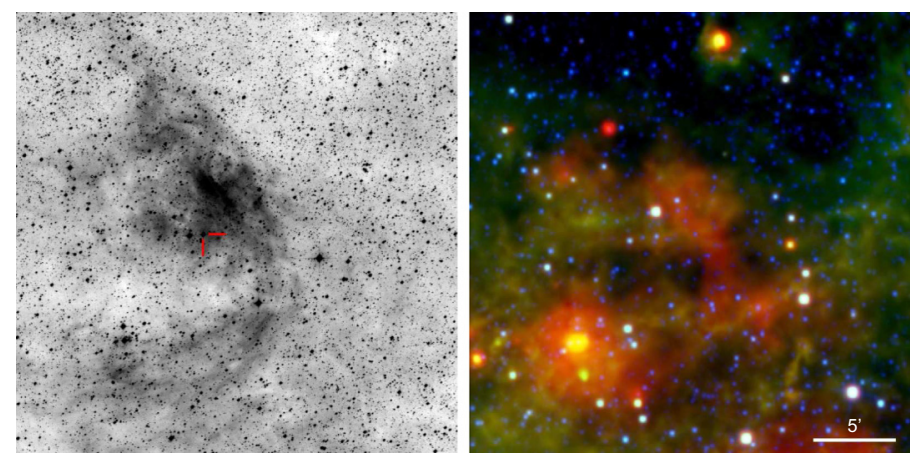

Fig. A.8. Same as Fig. A.1 for WR 55 (RCW 78).
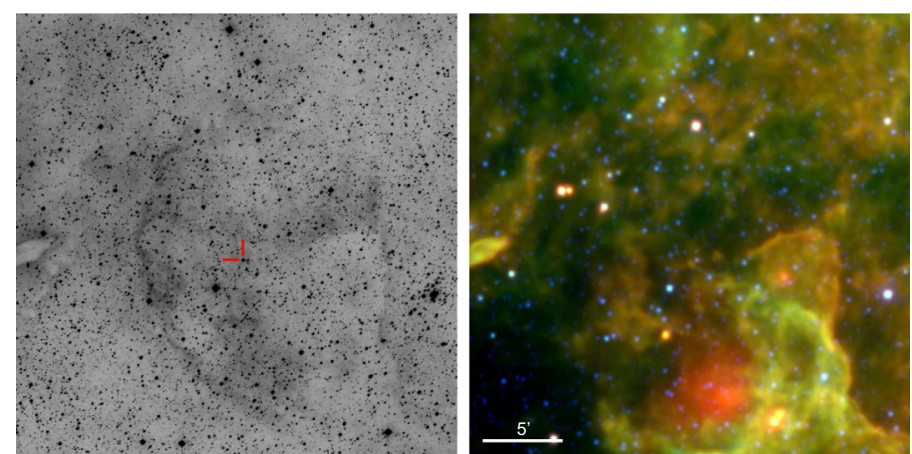

Fig. A.9. Same as Fig. A.1 for WR 68 (G320.5-1.4).
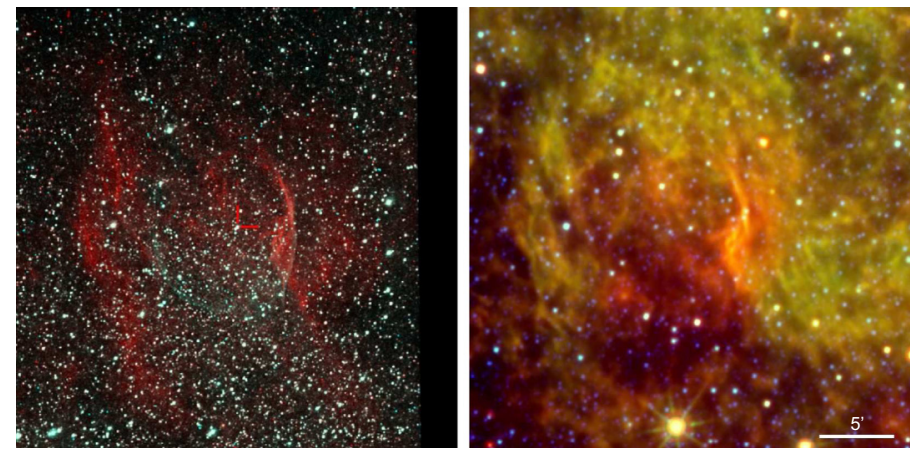

Fig. A.10. Same as Fig. A.1 for WR 75 (RCW 104).
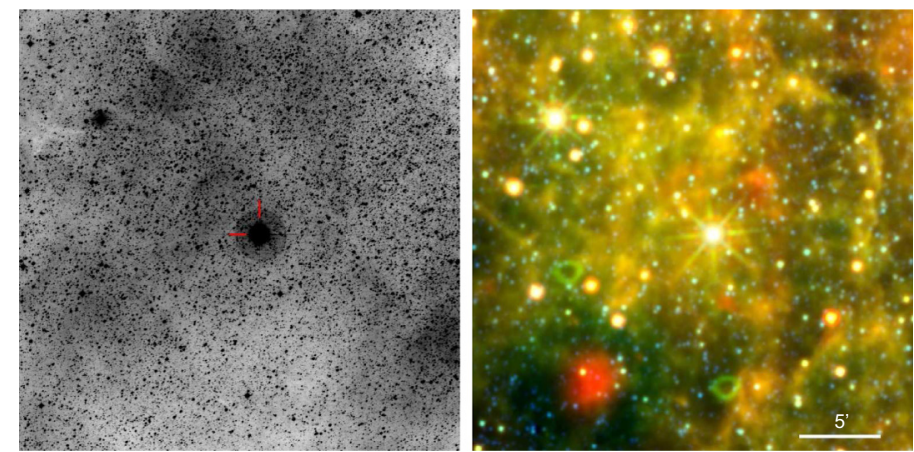

Fig. A.11. Same as Fig. A.1 for WR 85 (RCW 118).
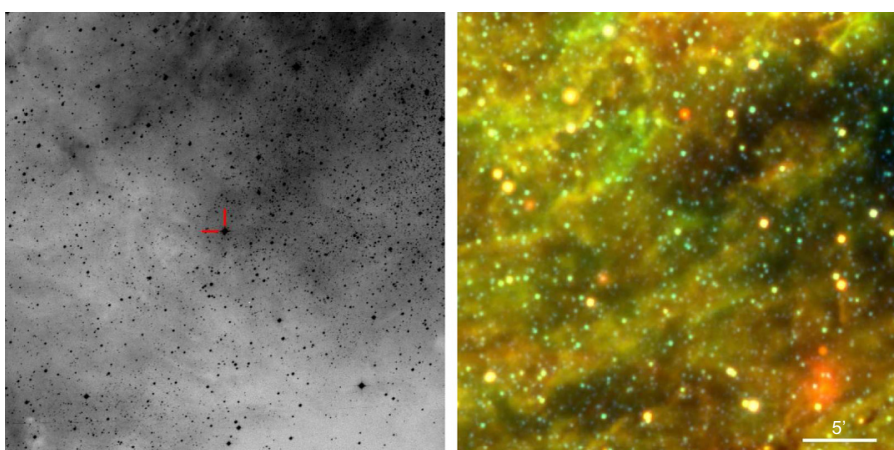

Fig. A.12. Same as Fig. A.1 for WR 86 (RCW 130).
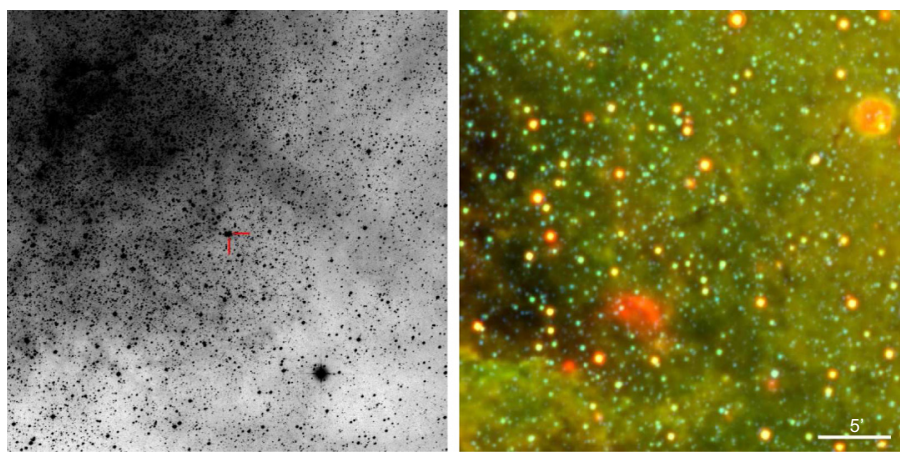

Fig. A.13. Same as Fig. A.1 for WR 94 (Anon).
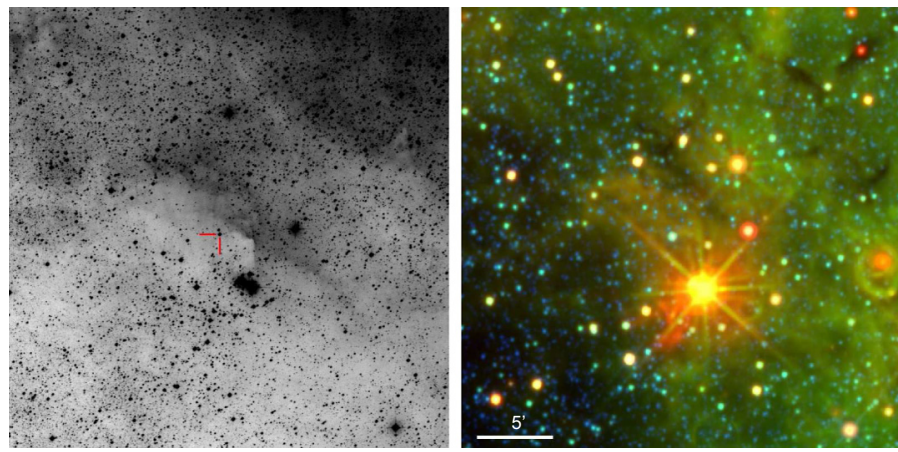

Fig. A.14. Same as Fig. A.1 for WR 95.
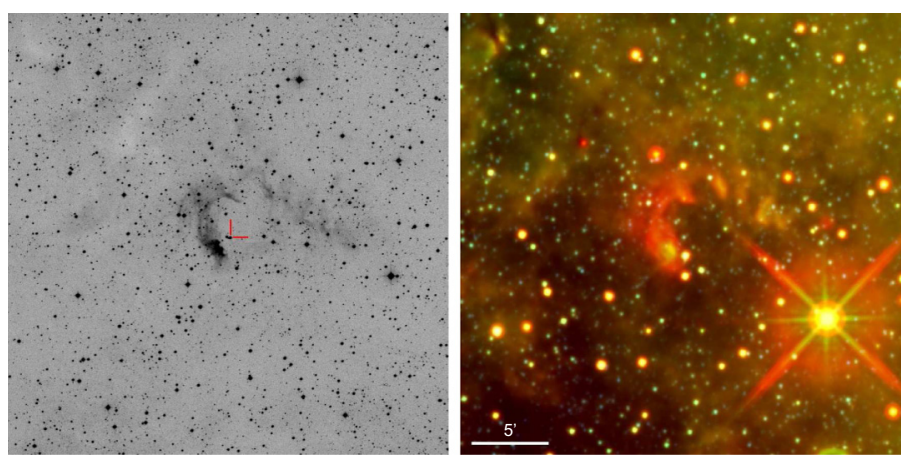

Fig. A.15. Same as Fig. A.1 for WR 101 (Anon). 
J. A. Toalá et al.: WISE morphological study of WR nebulae
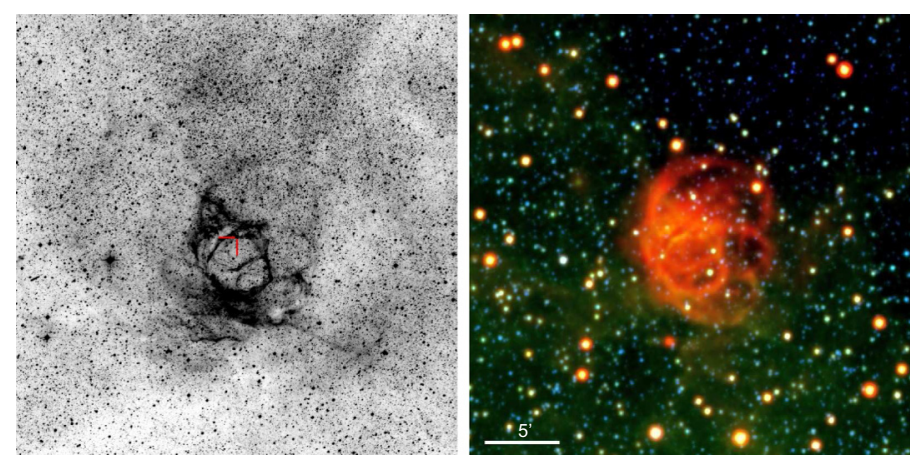

Fig. A.16. Same as Fig. A.1 for WR 102 (G2.4+1.4).
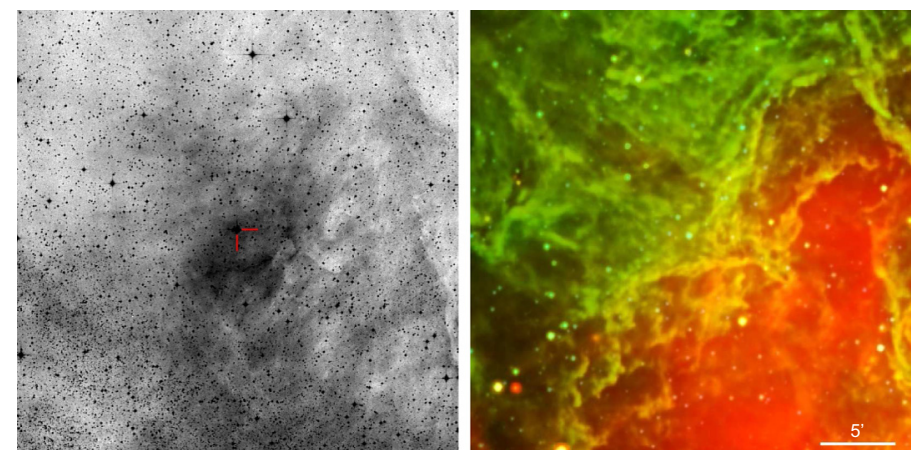

Fig. A.17. Same as Fig. A.1 for WR 113 (RCW 167).

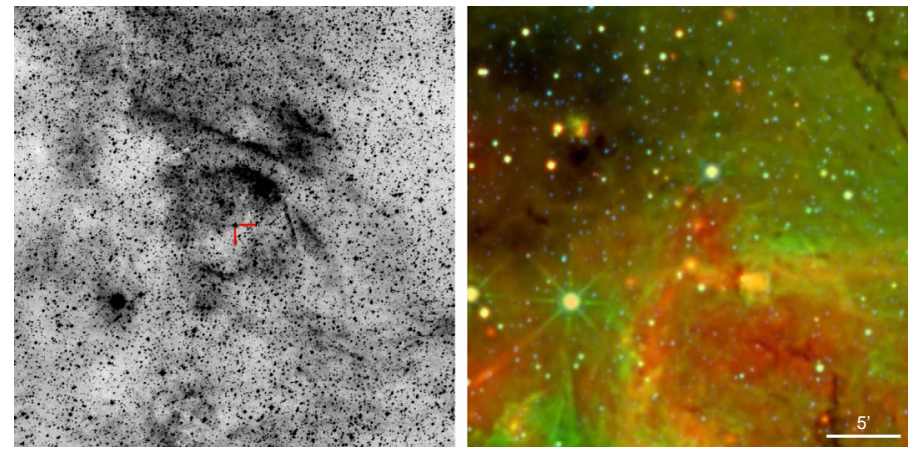

Fig. A.18. Same as Fig. A.1 for WR 116 (Anon).

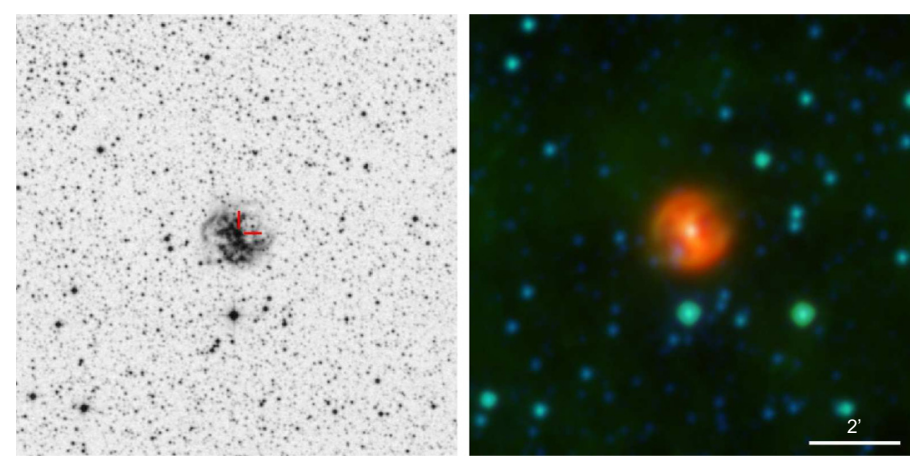

Fig. A.19. Same as Fig. A.1 for WR 124 (M1-67).
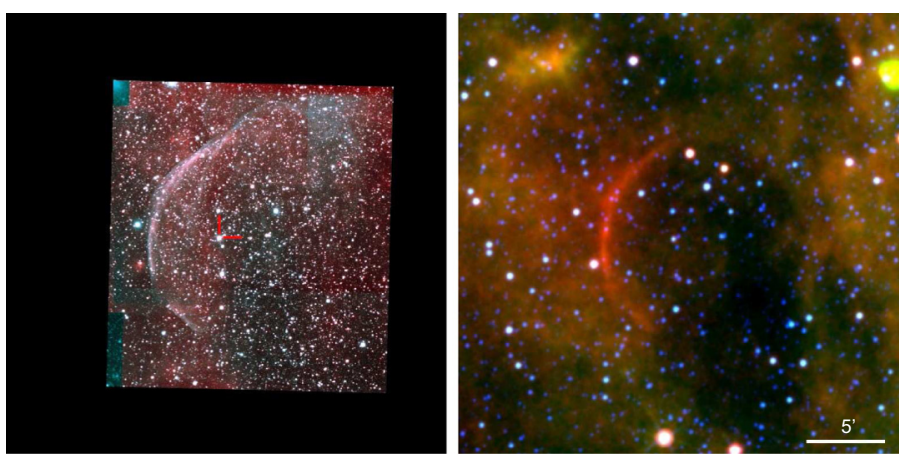

Fig. A.20. Same as Fig. A.1 for WR 128 (Anon).
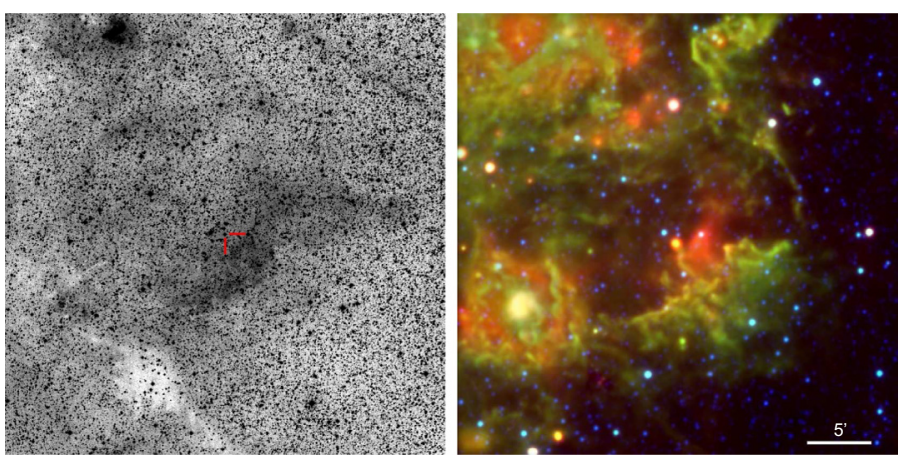

Fig. A.21. Same as Fig. A.1 for WR 131.
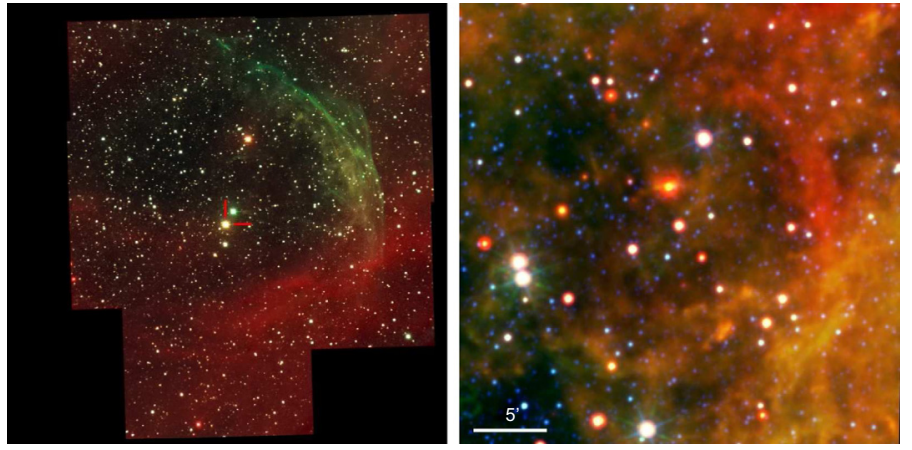

Fig. A.22. Same as Fig. A.1 for WR 134 (Anon).
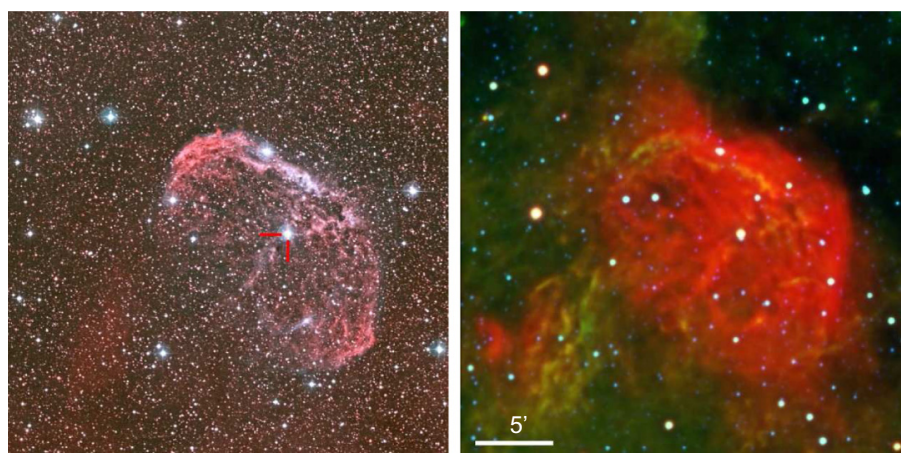

Fig. A.23. Same as Fig. A.1 for WR 136 (NGC 6888). 